En France l'Association des dames françaises, à côté du matériel envoyé en Russie pour un hòpital de 200 lits, a continué sa souscription en faveur des blessés russes de la guerre; au 20 novembre les sommes recueillies s'élevaient à fr. 42,647 40. La Socièté francaise de secours a, de sun côté, poursuivi l'aménagement de l'hòpital flottant l'Orel ${ }^{1}$. Tout y a été prévu avec un soin minutieux pour l'hopitalisation de 650 blessés ; l'installation a coúté fr. 250,000; en octobre 1904 elle était terminée et l'Orel attendait à Barcelone de se joindre à l'escadre de la Baltique. Le 12 novembre il rejoignait l'escadre à Tanger, salué avec un enthousiasme reconnaissant par un télégramme de l'amiral Rodjestventsky à l'ambassadrice de Russie à Paris, $\mathbf{M}^{\text {me }}$ de Nélidoff. La Croix-Rouge française a également offert, en témoignage de sa haute admiration pour son courage et son dévouement à la cause des blessés à Port-Arthur, les insignes en or de la Société à $M^{\text {me }}$ la générale Stcessel. Le total des dons au 15 novembre 1904, en faveur des victimes de la guerre, s'élevait à fr. 130,93975

La Société autrichienne de la Cronx-Rouge a décidé de mettre au service des Croix-Rouges du Japon et de la Russie une somme de 100,000 couronnes.

La Société hongroise a envoyé par voie diplomatique 10,000 couronnes à chacun des Comités de St-Pétersbourg et de Tokio.

Enfin le Comité international, dont les ressources sont exiguës, a envoyé, à titre de témoignage de sympathie, à la Société russe à Saint-Pétersbourg, ainsi qu'à la Croix-Rouge japonaise par l'obligeante entremise de la légation du Japon a Paris, la somme de fr. 1000 à chacune.

atTribution Des Revenus du Fonds aUgusta fT aPTEL POUR L'ACCROISSEMENT DE CE FONDS $112^{\mathrm{me}}$ circulaire aux Comités centruux.

Messieurs, Genève, 7 janvier 1905.

Appelé pour la seconde fois à attribuer à un Comité central de la Croix-Rouge les revenus du Fonds Augusta, en conformité de

${ }^{1}$ Voy. T. XXXV, p. 195. 
la décision prise à la Conférence de Saint-Pétersbourg, le Comité international s'est trouvé en présence de plusieurs demandes.

Devant les besoins sans cesse grandissants de l'assistance volontaire en Extrême-Orient, les efforts considérables faits par les Croix-Rouges des belligérants, secondées par celles des Etats neutres, pour apporter aux malades et blessés quelque soulagement à leurs souffrances et quelque atténuation de leurs maux, le Comité s'est senti pressé de faire contribuer à la réalisation de ce même buı humanitaire les revenus du Fonds Augusta ; c'était, il est vrai, une goutte d'eau dans l'océan des misères à adoucir, mais nous ne nous sommes pas sentis libres de la distraire et de l'employer ailleurs, persuadés que celle dont le Fonds porte le nom et de la mort de laquelle la date placée en tête de cette circulaire commémore l'anniversaire, eût, dans son cœur compatissant anx souffrances humaines, approuvé cette affectation.

Les revenus du Fonds Augusta en 1904 ont donc été attribués au Comité central des associations allemandes de la Croix-Rouge, qui en avait demandé l'allocation en faveur de l'œuvre d'assistance entreprise par lui tant en Mandchourie qu'au Japon.

En le faisant et en rendant ainsi indirectement hommage tant à la mémoire de l'impératrice Augusta qu'au Comité allemand lui-même, qui a contribué plus que tout autre à la constitution de ce Fonds, nous ne sortions pas des termes du règlement en vigueur. Cette allocation était bien consentie en vue de la réalisation d'un but d'utilité pratique. Tant à Yokohama qu'à Tschita, en Transbaïkalie, le Comité allemand a institué des lazarets admirablement outillés pour rendre le maximum de services à la cause des blessés militaires. A la tête du premier se trouve le $D^{r}$ Matthiolius, dont le nom est à juste titre connu depuis la guerre dı Transvaal, et qui, joignant la pratique à la science, saura enrichir la science médicale d'expériences précieuses. Le second, placé sous la direction du chirurgien $\mathrm{D}^{r}$ Brentano, a comme annexe un laboratoire bactério'ogique pourvo de loutes les installations nécessaires aux recherches scientifiques, et trouve, dans les épidémies de typhus et de choléra régnant aux environs, un riche champ d'expériences bactériologiques. La présence, à la tête de ce laboratoire, du $\mathrm{D}^{\mathrm{r}}$ Schutze, un élève de Koch, donne l'assurance que celte activité aura des résultats pratiques bienfaisants et utiles, non 
seulement pour les victimes de la guerre, mais aussi, d'une manière plus générale et plus durable, pour la cause universelle du soin des malades et des blessés.

Le Fonds Augusta se composait, au 31 décembre 1904, des mêmes valeurs qu'au 31 décembre $1903^{1}$. Les revenus de ce capital se sont montés en 1904 à fr. 3385 ; c'est donc cette somme qui a été allouée au Cornité central allemand.

Lorsque, dans sa séance plénière du 17 mai 1902, la Conférence de Saint-Pétersbourg a statué sur l'emploi du Fonds de l'impératrice Augusta, elle a approuvé en particulier un alinéa ainsi conçu :

"Un appel sera adressé aux amis de la Croix-Rouge, en tous pays, surtout à ceux des Comités centraux qui n'ont pas encore concouru à la formation de ce fonds, pour les engager à l'accroître ».

Or cette invitation, qui ne visait nominativement personne, n'a été exécutée par aucun des Comités centraux, ce qui n'a pas empêché cependant le Comilé central allemand de porter, ainsi qu'il l'avait annoncé, le capital de ce fonds à la somme de fr. 100,000 en chiffres ronds ${ }^{2}$. Le Comité international prend donc la liberié de relever cet appel et de lui donner la suite qu'il comporte.

Celte mesure lui paraît d'autant plus opportune que, maintenant, l'utilité du Fonds Augusta n'est plus hypothétique et que chaque année l'un des Comités centraux existants est appelé à en bénéficier. Malheureusement son aide est encore d'un bien faible secours à ceux qui en sollicitent l'appui et auxquels il est accordé car ses intérêts d'une seule année ne représentent qu'une somme presque dérisoire, en regard de l'importance des capitaux que réclame la Croix-Rouge pour pouvoir prendre tout son essor.

Nos honorables correspondants auxquel nous adressons ces lignes comprendront sans peine l'importance du présent appel et seront jaloux, nous voulons l'espérer, d'y répondre favorablement.

Veuillez agréer, Messieurs, l'assurance de nos sentiments les plus distingués.

Pour le Comité international de la Croix-Rouge:

Le Secrétaire,

E. ODIER.
Le Président,

G. MoYnier.

\section{Le Vice-Président,} Gustave ADOR.

1 Voy. T. XXXV, p. 9.

${ }^{2}$ Vog. T. XXXIV, p. 20. 